\title{
TECNOLOGIAS DO CUIDADO UTILIZADAS PELA ENFERMAGEM NA ASSISTÊNCIA AO PACIENTE POLITRAUMATIZADO: REVISÃO INTEGRATIVA
}

\author{
Virna Ribeiro Feitosa Cestari', Luís Rafael Leite Sampaio², Islene Victor Barbosa², Rita Mônica Borges Studart², Bruna Bárbara \\ Fernandes Moura ${ }^{1}$, Ana Rachel Cavalcante Araújo ${ }^{3}$
}

${ }^{1}$ Discente de Enfermagem. Universidade de Fortaleza. Fortaleza, CE, Brasil.

2Enfermeiro. Doutor em Enfermagem. Docente do Departamento de Enfermagem da Universidade de Fortaleza. Fortaleza, CE, Brasil.

${ }^{3}$ Enfermeira. Especialista em Enfermagem em Centro de Terapia Intensiva. Universidade Estadual do Ceará. Fortaleza, CE, Brasil.

RESUMO: Objetivou-se identificar as tecnologias do cuidado utilizadas pelo enfermeiro na assistência ao paciente politraumatizado. Revisão integrativa, com busca de artigos em três bases de dados, no período de maio a julho de 2014. Foram selecionados 19 artigos, compreendidos no período de 2009 a 2014, distribuídos nas três categorias tecnológicas do cuidado: leves, leve-duras e duras. Verificou-se que os profissionais de enfermagem utilizam os três tipos de tecnologias do cuidado na assistência ao paciente politraumatizado, com ênfase às leve-duras. Entre as tecnologias leves: apoio e educação em saúde do paciente e familiares/cuidadores e a capacitação da equipe de enfermagem; tecnologias leve-duras: gerência do cuidado, acolhimento do paciente com classificação de risco, avaliação e tratamento da dor, processo de enfermagem e elaboração de protocolos; e tecnologias duras: sistemas de informação. Percebeu-se a melhoria assistencial proporcionada pelas tecnologias do cuidado, por estas abrangerem todos os aspectos do cuidar.

DESCRITORES: Assistência pré-hospitalar; Traumatismo múltiplo; Cuidados de enfermagem; Enfermagem em emergência.

\section{HEALTHCARE TECHNOLOGIES USED IN NURSING TO CARE FOR POLYTRAUMATIZED PATIENTS: AN INTEGRATIVE REVIEW}

\section{TECNOLOGÍAS DEL CUIDADO UTILIZADAS POR LA ENFERMERÍA EN LA ASISTENCIA AL PACIENTE POLITRAUMATIZADO: REVISIÓN INTEGRATIVA}

\begin{abstract}
The aim of this study was to identify healthcare technologies used by nurses in the care for polytraumatized patients. This is an integrative review, with articles found in three databases in the period from May to July 2014 Nineteen articles from the period between 2009 and 2014 were selected and distributed among the three categories of healthcare technology: soft, soft-hard and hard. It was found that nursing workers adopted the three types of healthcare technology when caring for polytraumatized patients, emphasizing the soft-hard category. Soft technologies included health support and education of patient and family members/caregivers and training of the nursing team; soft-hard technologies: care management, admission of patients under risk, assessment and treatment of pain nursing process and development of protocols; and hard technologies: information systems. Care improvements caused by healthcare technologies were found, since they reach all aspects of care.
\end{abstract}

DESCRIPTORS: Prehospital care; Multiple trauma; Nursing care; Emergency nursing.
RESUMEN: El objetivo del estudio fue identificar las tecnologías del cuidado utilizadas por el enfermero en la asistencia al paciente politraumatizado. Revisión integrativa, cuya búsqueda de artículos ocurrió en tres bases de datos, en el periodo de mayo a julio de 2014. Fueron seleccionados 19 artículos, comprendidos en el periodo de 2009 a 2014, distribuidos en las tres categorías tecnológicas de cuidado: leves, leve-duras y duras. Se verificó que los profesionales de enfermería utilizan los tres tipos de tecnologías del cuidado en la asistencia al paciente politraumatizado, con énfasis a las leve-duras. Entre las tecnologías leves: apoyo y educación en salud del paciente y familiares/cuidadores y la capacitación del equipo de enfermería; tecnologías leve-duras: gerencia del cuidado, acojimiento del paciente con clasificación de riesgo, evaluación y tratamiento del dolor, proceso de enfermería y elaboración de protocolos; y tecnologías duras: sistemas de información. Se percibió la mejoría asistencial proporcionada por las tecnologías del cuidado, a causa de que estas abarcan todos los aspectos del cuidar.

DESCRIPTORES: Asistencia prehospitalar; Traumatismo múltiplo; Cuidados de enfermería; Enfermería en emergencia. 


\section{INTRODUÇÃO}

O politraumatismo advém de um evento traumático em que há grande desprendimento de energia, como quedas, acidentes de trânsito, atropelamentos e ferimentos por armas de fogo, entre outras causas que resultem em graves lesões $^{(1)}$. É considerado a primeira causa de morte entre os indivíduos na faixa etária de 20 a 40 anos de idade, ou seja, na fase em que o indivíduo é mais produtivo, sendo as vítimas, na grande maioria, do sexo masculino. Sua incidência tem preocupado pesquisadores e gestores, por gerarem impactos econômicos e sociais ${ }^{(2)}$.

As lesões relacionadas ao trauma podem ocasionar incapacidades físicas e/ou mentais, temporárias ou permanentes e também levar ao óbito ${ }^{(3)}$. O indivíduo vítima de trauma passa por um processo doloroso, que inclui confusão, medo pelo desconhecido e temor frente à morte, a mutilação, imobilização e outras alterações na sua identidade e integridade corporal, advindas como efeitos do trauma, que pode, ainda, afetar sua capacidade de autocuidado ${ }^{(4)}$.

A necessidade de individualizar a assistência é realidade vivenciada por todos os profissionais que participam desses atendimentos, devido às diferentes formas de apresentação, gravidade e complexidade dos traumas ${ }^{(5)}$. O enfermeiro tem papel fundamental na assistência à vítima de trauma, pois, como coordenador da equipe de enfermagem, deve programar e priorizar a assistência a ser prestada e estabelecer medidas preventivas e reparadoras, em um cenário em que o tempo entre a vida e a morte é tênue ${ }^{(6)}$. Para tal, destaca-se a importância da produção de tecnologia para as inovações na área da saúde e enfermagem, pois auxilia a organização do serviço, as formas de cuidados, fundamenta e inova a prática do exercício profissional( ${ }^{(7)}$.

As tecnologias do cuidado são divididas em dura, leve-dura e leve. Tecnologia dura ou trabalho morto refere-se ao instrumental complexo em seu conjunto, englobando todos os equipamentos para tratamentos, exames e a organização das informações; a leve-dura refere-se aos saberes profissionais, bem estruturados com a clínica, a epidemiologia e os demais profissionais que compõem a equipe. As tecnologias leves são aquelas utilizadas nas relações humanas, como a produção de vínculo, autonomização, acolhimento, no encontro entre o profissional e o usuário/paciente ${ }^{(8)}$.

\section{A compreensão do cuidado clínico}

em enfermagem em emergência a partir das tecnologias permite a construção da responsabilização, confiabilidade, relações de vínculo e acolhimento. Nessa perspectiva, o cuidado de enfermagem deve buscar a harmonização destas três dimensões de tecnologias, determinantes para a qualidade do cuidado $^{(9)}$.

Diante da responsabilidade do enfermeiro junto aos pacientes vítimas de trauma e conhecendo-se a complexidade e especificidades do atendimento a esses indivíduos, que necessitam cada vez mais de uma maior atenção do enfermeiro, despertou-se acerca da busca de publicações de enfermagem que envolvam o uso de tecnologias de cuidados dispensadas a esta clientela.

Este estudo torna-se relevante para a enfermagem em urgência e emergência por fornecer subsídios para a prática clínica e por evidenciar a necessidade da reconstrução de um modelo assistencial que tenha como objetivo o cuidado ao paciente politraumatizado em toda sua magnitude. Pretende-se, também, a divulgação das produções científicas disponíveis para a avaliação de opções e tomada de decisão no cuidado a estes pacientes, mediante a aplicabilidade das tecnologias.

Com base na realidade descrita, objetivou-se identificar as tecnologias do cuidado utilizadas pelo enfermeiro na assistência ao paciente politraumatizado.

\section{MÉTODO}

Trata-se de uma revisão integrativa da literatura, método que tem como finalidade sintetizar resultados obtidos em pesquisas sobre um tema ou questão, de maneira sistemática, ordenada e abrangente, por meio do cumprimento criterioso de seis etapas: identificação da questão da pesquisa; definição das características das pesquisas primárias da amostra; seleção, por pares, das pesquisas que compuseram a amostra; análise dos achados dos artigos; interpretação dos resultados e relato da revisão, proporcionando um exame crítico dos achados ${ }^{(10)}$.

No intuito de guiar esta revisão, formulou-se a seguinte questão norteadora: Quais as tecnologias do cuidado que o enfermeiro tem utilizado na assistência ao paciente politraumatizado?

A busca foi realizada no período de maio a junho de 2014, utilizando-se as seguintes bases 
de dados: Literatura Latino-Americana e do Caribe em Ciências da Saúde (LILACS), Scientific Eletronic Library Online (SciELO) e Base de Dados da Enfermagem (BDENF), com base nos Descritores em Ciências da Saúde: "traumatismo múltiplo", "enfermagem em emergência" e "cuidados de enfermagem". A equação de busca foi ("traumatismo múltiplo" AND "enfermagem em emergência") OR ("traumatismo múltiplo" AND "cuidados de enfermagem").

Os critérios de inclusão das publicações selecionadas para o estudo foram: artigos que contemplassem o objetivo proposto; indexadas nas bases de dados LILACS, SciELO e BDENF; publicadas no período de 2009-2014; nos idiomas português, inglês e espanhol; e disponíveis eletronicamente na íntegra. Foram excluídos os editoriais, cartas ao editor, trabalhos publicados em anais de evento, artigos de reflexão e publicações duplicadas.

A partir do cruzamento dos descritores, foram localizados 112 artigos na LILACS, 86 na SciELO e 16 na BDENF. Para seleção da amostra, efetuaramse três etapas. A primeira deu-se pela leitura dos títulos dos artigos, sendo excluídos os que não tivessem relação com o tema. Posteriormente, foram lidos os resumos, com o intuito de uma maior aproximação e conhecimento do trabalho. Após essa seleção, buscaram-se os textos que se encontravam disponíveis na íntegra.

O corpus da revisão integrativa foi composto por 19 artigos, tabulados segundo as seguintes categorias: título, periódico e ano de publicação, tipo de estudo e nível de evidência. Para facilitar o alcance do objetivo deste estudo, as informações foram consolidadas por categorização dos dados, os quais foram divididos de acordo com as tecnologias do cuidado utilizadas pelos enfermeiros na assistência às vítimas de trauma.

Para a classificação do nível de evidências (NE), considerou-se: nível I - as evidências são provenientes de revisão sistemática de estudos randomizados controlados ou oriundas de revisões sistemáticas de ensaios clínicos randomizados; nível II - evidências derivadas de revisões sistemáticas randomizadas individuais ou observacionais; nível III - evidências obtidas de estudos sem randomização controlados, coorte ou de seguimento; nível IV - evidências provenientes de caso-controle bem delineados, estudos de caso e longitudinais; nível V evidências originárias de estudos descritivos ${ }^{(11)}$.

\section{RESULTADOS}

Os estudos foram divididos em quadros, de acordo com o tipo de tecnologia utilizada e organizados quanto ao ano de publicação: do mais recente ao mais antigo.

Os países de origem da publicação dos estudos que compuseram a amostra foram Brasil, com 17 (89,5\%), México e Colúmbia, com um $(10,5 \%)$ cada. Em análise aos estados e regiões de publicação dos artigos nacionais, constatou-se o predomínio de estudos oriundos do eixo SulSudeste, evidenciando a hegemonia da produção científica dos enfermeiros destas regiões, com destaque para Santa Catarina, com cinco $(26,3 \%)$ estudos, e São Paulo, com oito (42,1\%).

Em relação aos anos de publicação dos artigos integrantes do estudo, constatou-se que, no

Quadro 1 - Resultados dos artigos selecionados de acordo com a utilização das tecnologias leves na assistência ao paciente politraumatizado. Fortaleza, CE, Brasil, 2014

\begin{tabular}{|c|c|c|c|}
\hline Título & $\begin{array}{l}\text { Periódico/ano de } \\
\text { publicação }\end{array}$ & $\begin{array}{l}\text { Tipo/abordagem } \\
\text { do estudo }\end{array}$ & $\begin{array}{l}\text { Nível de } \\
\text { evidência }\end{array}$ \\
\hline $\begin{array}{l}\text { Percepção dos profissionais de enfermagem frente à } \\
\text { identificação, quantificação e tratamento da dor em } \\
\text { pacientes de uma unidade de terapia intensiva de trauma }\end{array}$ & $\begin{array}{l}\text { Revista Dor, } \\
2011\end{array}$ & $\begin{array}{l}\text { Prospectivo e } \\
\text { quantitativo }\end{array}$ & IV \\
\hline El cuidado de enfermería significa ayuda & Aquichan, 2010 & $\begin{array}{l}\text { Exploratório, } \\
\text { qualitativo }\end{array}$ & V \\
\hline $\begin{array}{l}\text { Enseñar a quien cuida el arte de cuidar: un programa educativo } \\
\text { con cuidadores de pacientes politraumatizados }\end{array}$ & $\begin{array}{l}\text { Investigación y } \\
\text { Educación em } \\
\text { Enfermería, } 2010\end{array}$ & $\begin{array}{l}\text { Longitudinal, } \\
\text { de intervenção, } \\
\text { quantitativo }\end{array}$ & IV \\
\hline $\begin{array}{l}\text { A capacitação da equipe que atua no atendimento pré- } \\
\text { hospitalar móvel: necessidade e importância da educação } \\
\text { permanente na perspectiva dos trabalhadores }\end{array}$ & $\begin{array}{l}\text { Revista Mineira } \\
\text { de Enfermagem, } \\
2009\end{array}$ & $\begin{array}{l}\text { Descritivo e } \\
\text { exploratório, } \\
\text { qualitativo }\end{array}$ & V \\
\hline
\end{tabular}


Quadro 2 - Resultados dos artigos selecionados de acordo com a utilização das tecnologias leve-duras na assistência ao paciente politraumatizado. Fortaleza, Ceará, Brasil, 2014

\begin{tabular}{|c|c|c|c|}
\hline Título & $\begin{array}{l}\text { Periódico/ano de } \\
\text { publicação }\end{array}$ & $\begin{array}{l}\text { Tipo/ } \\
\text { abordagem de } \\
\text { estudo }\end{array}$ & $\begin{array}{l}\text { Nível de } \\
\text { evidência }\end{array}$ \\
\hline $\begin{array}{l}\text { Acolhimento com avaliação e classificação de risco: } \\
\text { concordância entre os enfermeiros e o protocolo } \\
\text { institucional }\end{array}$ & $\begin{array}{l}\text { Revista Latino- } \\
\text { Americana de } \\
\text { enfermagem, } 2013\end{array}$ & $\begin{array}{l}\text { Descritivo, } \\
\text { quantitativo }\end{array}$ & $\mathrm{V}$ \\
\hline $\begin{array}{l}\text { Diagnósticos reais e propostas de intervenções de } \\
\text { enfermagem para os pacientes vítimas de múltiplos } \\
\text { traumas }\end{array}$ & $\begin{array}{l}\text { Revista Eletrônica de } \\
\text { Enfermagem, } 2013\end{array}$ & $\begin{array}{l}\text { Descritivo, } \\
\text { quantitativo }\end{array}$ & $\mathrm{V}$ \\
\hline $\begin{array}{l}\text { Desafios para a gerência do cuidado em emergência na } \\
\text { perspectiva de enfermeiros }\end{array}$ & $\begin{array}{l}\text { Acta Paulista de } \\
\text { Enfermagem, } 2013\end{array}$ & $\begin{array}{l}\text { Descritivo e } \\
\text { exploratório, } \\
\text { qualitativo }\end{array}$ & $\mathrm{V}$ \\
\hline $\begin{array}{l}\text { Acolhimento com classificação de risco em serviço } \\
\text { hospitalar de emergência: avaliação da equipe de } \\
\text { enfermagem }\end{array}$ & $\begin{array}{l}\text { Revista Mineira de } \\
\text { Enfermagem, } 2012\end{array}$ & $\begin{array}{l}\text { Transversal, } \\
\text { quantitativo }\end{array}$ & III \\
\hline $\begin{array}{l}\text { Avaliação do indivíduo vítima de politraumatismo pela } \\
\text { equipe de enfermagem em um serviço de emergência de } \\
\text { Santa Catarina }\end{array}$ & $\begin{array}{l}\text { Revista Brasileira de } \\
\text { Promoção da Saúde, } \\
2012\end{array}$ & \begin{tabular}{|l|} 
Estudo \\
de caso, \\
qualitativo \\
\end{tabular} & IV \\
\hline $\begin{array}{l}\text { Diagnósticos de enfermagem em vítimas fatais decorrentes } \\
\text { de trauma no cenário da emergência }\end{array}$ & $\begin{array}{l}\text { Revista Latino- } \\
\text { Americana de } \\
\text { Enfermagem, } 2012\end{array}$ & $\begin{array}{l}\text { Transversal, } \\
\text { descritivo e } \\
\text { exploratório, } \\
\text { quantitativo }\end{array}$ & III \\
\hline $\begin{array}{l}\text { Diagnósticos de enfermagem em vítimas de trauma nas } \\
\text { primeiras seis horas após o evento }\end{array}$ & $\begin{array}{l}\text { Acta Paulista de } \\
\text { Enfermagem, } 2012\end{array}$ & $\begin{array}{l}\text { Prospectivo } \\
\text { transversal, } \\
\text { quantitativo }\end{array}$ & III \\
\hline $\begin{array}{l}\text { A multidimensionalidade da dor no ensino de enfermagem } \\
\text { em atendimento pré-hospitalar às vítimas de trauma }\end{array}$ & Revista Dor, 2012 & $\begin{array}{l}\text { Descritivo e } \\
\text { exploratório, } \\
\text { qualitativo }\end{array}$ & $\mathrm{V}$ \\
\hline $\begin{array}{l}\text { Classificação de risco na emergência: avaliação da equipe } \\
\text { de enfermagem }\end{array}$ & $\begin{array}{l}\text { Revista de } \\
\text { Enfermagem da } \\
\text { UERJ, } 2011 \\
\end{array}$ & $\begin{array}{l}\text { Descritivo, } \\
\text { qualitativo }\end{array}$ & $\mathrm{V}$ \\
\hline $\begin{array}{l}\text { Protocolo de cuidados de enfermagem no ambiente } \\
\text { aeroespacial a pacientes traumatizados: cuidados antes do } \\
\text { voo }\end{array}$ & $\begin{array}{l}\text { Revista Brasileira de } \\
\text { Enfermagem, } 2011\end{array}$ & $\begin{array}{l}\text { Pesquisa } \\
\text { qualitativa, } \\
\text { convergente } \\
\text { assistencial } \\
\end{array}$ & $\mathrm{V}$ \\
\hline $\begin{array}{l}\text { O enfermeiro no cuidado à vítima de trauma com dor: o } \\
\text { quinto sinal vital }\end{array}$ & $\begin{array}{l}\text { Revista Escola de } \\
\text { Enfermagem da USP, } \\
2011\end{array}$ & $\begin{array}{l}\text { Exploratório, } \\
\text { qualitativo }\end{array}$ & $\mathrm{V}$ \\
\hline $\begin{array}{l}\text { Percepção da equipe de enfermagem de um serviço de } \\
\text { atendimento pré-hospitalar móvel sobre o gerenciamento } \\
\text { de enfermagem }\end{array}$ & $\begin{array}{l}\text { Texto e Contexto } \\
\text { Enfermagem, } 2010\end{array}$ & $\begin{array}{l}\text { Exploratório } \\
\text { e qualitativo }\end{array}$ & $\mathrm{V}$ \\
\hline $\begin{array}{l}\text { Diagnósticos de enfermagem em vítimas de trauma } \\
\text { atendidas em um serviço pré-hospitalar avançado móvel }\end{array}$ & $\begin{array}{l}\text { Revista Eletrônica de } \\
\text { Enfermagem, } 2009\end{array}$ & $\begin{array}{l}\text { Exploratório } \\
\text { e quantitativo }\end{array}$ & $\mathrm{V}$ \\
\hline
\end{tabular}

período de recorte, o ano de 2012 destacou-se com o maior quantitativo de pesquisas $(26,3 \%)$. Destacaram-se importantes revistas nacionais e internacionais, dentre as quais merece evidência a Revista Eletrônica de Enfermagem, com três publicações $(15,8 \%)$. No que concerne às modalidades dos estudos, ressalta-se que todos os artigos são originais, com destaque para os estudos exploratórios (26,3\%). Com relação à abordagem dos estudos, houve predomínio da quantitativa $(52,6 \%)$.

Das publicações $(68,4 \%)$ apresentou NE V; seguido do NE III e IV, com três (15,8\%) artigos. Em análise à utilização das tecnologias do cuidado na assistência de enfermagem ao paciente politraumatizado, verificou-se o predomínio das tecnologias leve-duras, abordadas em 13 (68,4\%) publicações, seguidas das leves e das duras, conforme exposto na Figura 1. 
Quadro 3 - Resultados dos artigos selecionados de acordo com a utilização das tecnologias duras na assistência ao paciente politraumatizado. Fortaleza, Ceará, Brasil, 2014

\begin{tabular}{|l|l|l|c|}
\hline Título & $\begin{array}{l}\text { Periódico/ano de } \\
\text { publicação }\end{array}$ & $\begin{array}{l}\text { Tipo/ } \\
\text { abordagem } \\
\text { de estudo }\end{array}$ & $\begin{array}{l}\text { Nível de } \\
\text { evidência }\end{array}$ \\
\hline $\begin{array}{l}\text { Diagnósticos e intervenções de Enfermagem em vítimas } \\
\text { de trauma durante atendimento pré-hospitalar utilizando a } \\
\text { CIPE }\end{array}$ & $\begin{array}{l}\text { Revista Eletrônica de } \\
\text { Enfermagem, 2013 }\end{array}$ & $\begin{array}{l}\text { Descritivo, } \\
\text { quantitativo }\end{array}$ & $\mathrm{V}$ \\
\hline $\begin{array}{l}\text { Sistema de auxílio aos diagnósticos de enfermagem para } \\
\text { vítimas de trauma no atendimento avançado pré-hospitalar } \\
\text { móvel utilizando as Taxonomias NANDA e NIC }\end{array}$ & $\begin{array}{l}\text { Journal of Health } \\
\text { Informatics, 2010 }\end{array}$ & $\begin{array}{l}\text { Exploratório } \\
\text { e } \\
\text { quantitativo }\end{array}$ & $\mathrm{V}$ \\
\hline
\end{tabular}

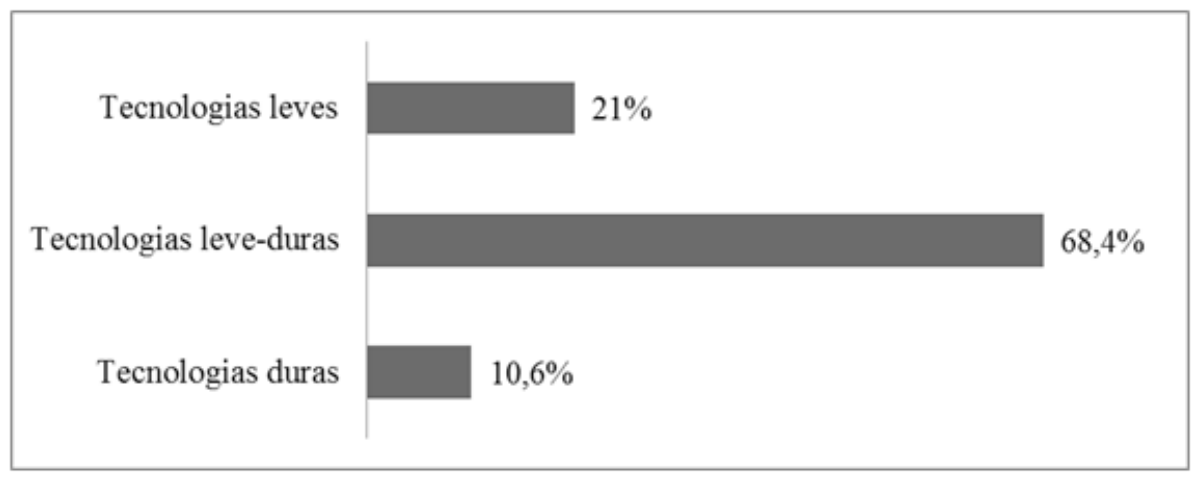

Figura 1. Quantitativo de artigos referentes a cada tecnologia do cuidado utilizada pela enfermagem na assistência a pacientes politraumatizados. Fortaleza, CE, Brasil, 2014

A análise da temática possibilitou categorizar, interpretar e agrupar os dados semelhantes. Desta forma, o agrupamento permitiu a síntese do conhecimento contemplado na literatura, dividida de acordo com as tecnologias de cuidado utilizadas pelos enfermeiros e sua equipe.

\section{DISCUSSÃO}

Verificou-se um aumento de publicações relacionadas com a utilização das tecnologias do cuidado na prática assistencial do enfermeiro nos anos analisados. No processo do trabalho de enfermagem constataram-se importantes articulações do cuidado mediante a utilização das tecnologias.

Entre os estudos, observou-se o predomínio do delineamento quantitativo, abordagem que objetiva trazer à luz dados, indicadores e tendências observáveis, gerando medidas confiáveis e sem vieses ${ }^{(12)}$. Entre os países de origem das pesquisas, o Brasil encontra-se na primeira colocação em termos de publicação, o que sugere um interesse nacional no estudo da temática.

\section{Tecnologias de cuidado leves}

As tecnologias leves ou relacionais se fazem necessárias no entorno da ação de cuidar(8). $\mathrm{Na}$ literatura consultada para a elaboração desta revisão, as tecnologias leves utilizadas pelos enfermeiros foram a de apoio e educação em saúde do paciente e familiar, bem como a capacitação da equipe de enfermagem.

O cuidado implica em um compromisso por parte da equipe de enfermagem para com os pacientes. Esse cuidado envolve a gestão de tecnologia, a capacidade de prevenir complicações, à adoção de comportamentos e atitudes de apoio destinadas a dignificar os outros na sua condição humana e proporcionar crescimento global ${ }^{(1-2)}$.

Devido às alterações e sequelas oriundas do trauma, verifica-se a necessidade de preparo do enfermeiro para o gerenciamento do cuidado ao paciente politraumatizado, que abrange desde a supervisão e capacitação da equipe de enfermagem, ao conforto físico e emocional, a escuta terapêutica e o cuidado humanizado ${ }^{(4)}$.

Pesquisa recente verificou a importância em se compreender como os indivíduos vivenciaram a experiência de uma situação de trauma. $\mathrm{O}$ estudo discriminou os sentimentos $\mathrm{e}$ as 
emoções vividas durante o trauma em categorias: emoções de fundo (ansiedade, preocupação, desespero), primárias ou universais (medo e tristeza) e secundárias (culpa). As categorias relações interpessoais foram: atendimento (apreciação geral) e relacionamento (relação com os profissionais). Os autores afirmaram que, categorizando informações, o enfermeiro pôde exercer ações integrais e holísticas, essenciais para o cuidado ${ }^{(13)}$.

A capacitação para o cuidado é de extrema importância para a realização de uma assistência efetiva. As ações educativas em saúde podem capacitar indivíduos e grupos na construção de novos conhecimentos, conduzindo a uma prática consistente de comportamentos preventivos ou de promoção da saúde ${ }^{(4)}$. Dados de um estudo de intervenção que buscou avaliar a efetividade de um programa de intervenção educativa dirigido a cuidadores de pacientes politraumatizados, evidenciou que, esse tipo de medida educativa tanto melhorou o nível de conhecimento dos cuidados, como os pacientes apresentaram menor incidência de complicações secundárias à imobilidade prolongada ${ }^{(14)}$.

Outra pesquisa evidenciou a importância da capacitação da equipe que atua no atendimento pré-hospitalar móvel. A capacitação foi considerada necessária para que o profissional se sentisse seguro e apto para prestar o socorro, habilitando o mesmo para prestar uma assistência com coerência, rapidez e segurança a diversidade de situações de trauma ${ }^{(15)}$.

\section{Tecnologias de cuidado leve-duras}

As tecnologias leve-duras envolvem todo o conhecimento e metodologias assistenciais na execução do cuidado ${ }^{(8)}$. Na assistência ao paciente politraumatizado, as tecnologias leve-duras utilizadas foram: gerência do cuidado, avaliação e acolhimento do paciente com classificação de risco (ACR), avaliação e tratamento da dor, processo de enfermagem (levantamento dos diagnósticos e implantação das intervenções de enfermagem) e elaboração de protocolos.

Os cuidados de enfermagem ao paciente vítima de trauma tem início com gerenciamento do serviço e da assistência, visto que estas são prerrogativas que qualificam o enfermeiro como coordenador do setor de urgência e emergência ${ }^{(5)}$.

Dentre as competências do enfermeiro no Atendimento Pré-Hospitalar (APH) estão: supervisionar e avaliar as ações da equipe de enfermagem; executar prescrições médicas por telemedicina; prestar cuidados de enfermagem de maior complexidade técnica a pacientes graves e com risco de morte; e capacidade de tomar decisões imediatas ${ }^{(16)}$.

Percebe-se, portanto, que o gerenciamento do enfermeiro no APH é uma atividade essencial, especialmente pelo grau de complexidade das ações, bem como pelas características peculiares desse serviço. $O$ enfermeiro destaca-se no processo de administração e gerenciamento de técnicas e recursos materiais. Este último justificase pelo fato da assistência não poder sofrer interrupções, seja pela falta ou pela má qualidade de determinado material, especialmente de emergência ou urgência ${ }^{(9)}$.

Estudo aponta para desafios dos enfermeiros na gerência do cuidado em emergência, como superlotação, manutenção da qualidade do cuidado e utilização da liderança como instrumento gerencial ${ }^{(17)}$. Verifica-se, portanto, a necessidade de alteração no fluxo de atendimento, tornando essencial um sistema de avaliação e ACR.

O ACR caracteriza-se por um processo dinâmico de identificação dos pacientes que necessitam de tratamento imediato, de acordo com o potencial de risco. É uma postura ética e profissional para o atendimento por nível de complexidade, mediante uso de escalas e protocolos que estratificam o risco em níveis. As mais utilizadas são: Emergency Severity Índex (ESI), Australian Triage Scale (ATS), Canadian Triage Acuity Scale (CTAS) e Manchester Triage System (MTS) ${ }^{(18)}$.

Autores corroboram que a ACR permite atendimento mais rápido e humano aos usuários com agravos agudos de saúde. Quanto às fragilidades, apontam a deficiência do espaço físico, materiais e de recursos humanos, e sinalizam a inexistência de referência e contrarreferência, com consequente acúmulo de usuários no serviço, interferindo na qualidade do atendimento assim como na satisfação dos profissionais do serviço de emergência ${ }^{(19-21)}$.

A avaliação pela equipe de enfermagem inicia-se com a realização do exame primário (Vias aéreas com proteção da coluna cervical, Respiração e ventilação, Circulação com controle da hemorragia, Incapacidade/estado neurológico e Exposição/controle do ambiente - ABCDE), e secundário, com avaliação minuciosa da vítima de politraumatismo ${ }^{(1)}$. A avaliação minuciosa permite a investigação da intensidade da dor, que causa prejuízos significativos ao paciente, como 
aumento da pressão arterial e frequência cardíaca, aumento do sangramento, aumento da sudorese, palidez, diminuição da oferta de oxigênio aos músculos, hipoventilação, hipóxia e respiração superficial. Autores corroboram que a dor deve ser avaliada no momento do atendimento $\mathrm{e}$ reavaliada em intervalos curtos. Desse modo, os profissionais identificarão a magnitude da variação da queixa álgica, possibilitando a realização de ajustes terapêuticos ${ }^{(22)}$.

Para o alívio da dor, destacam-se as medidas farmacológicas, não-farmacológicas e combinadas. No que diz respeito às medidas farmacológicas, é a equipe de enfermagem que programa a terapia farmacológica prescrita. Dessa forma, medicar o paciente implica em conhecer, além das vias de administração dos fármacos, sua indicação, ação farmacológica, reações, posologia e interações medicamentosas ${ }^{(23)}$.

No tocante às medidas não-farmacológicas, estudos evidenciam que fazem parte das atividades do enfermeiro para alívio da dor no paciente com trauma: distração; educação; técnicas de relaxamento e aplicação do calor ou frio, para reduzia a dor; medidas de conforto e higiene; massagens; apoio e tranquilização; posicionamento adequado; e controle de fatores ambientais ${ }^{(22-23)}$.

Outra tecnologia leve-dura de grande importância no cuidado ao paciente vítima de trauma é a utilização do processo de enfermagem. Os diagnósticos de enfermagem são um julgamento clínico sobre as respostas do indivíduo, da família ou da comunidade a problemas de saúde/processos vitais reais ou potenciais. Eles proporcionam a sustentação para a seleção de intervenções de enfermagem, a fim de atingir resultados pelos quais a enfermagem é responsável ${ }^{(5)}$.

Verificou-se através da literatura que a principal abordagem para a identificação dos diagnósticos de enfermagem compreendeu na realização do exame físico minucioso e pontual, direcionado para o comprometimento do sistema envolvido e suas complicações ${ }^{(1)}$. Encontraram-se quatro publicações acerca dos diagnósticos de enfermagem aos pacientes vítimas de trauma. Foram incluídos neste estudo os diagnósticos presentes em mais de $50 \%$ da casuística nos artigos selecionados.

Em estudo realizado com 23 pacientes adultos vítimas de trauma, verificou-se o levantamento de 24 diagnósticos de enfermagem, com predomínio:
Risco para infecção (91,3\%); Risco para trauma $(82,6 \%)$; Dor aguda (73,9\%); e Integridade tissular prejudicada $(65,2 \%)^{(3)}$.

Outro estudo, realizado com 406 vítimas, os autores fizeram olevantamento de 42 diagnósticos, sendo os mais frequentes: Risco para infecção $(95,4 \%)$; Risco de aspiração $(86,4 \%)$; Integridade da pele prejudicada $(84 \%)$; Padrão respiratório ineficaz $(82,8 \%)$; Risco de sangramento $(79,5 \%)$; Ventilação espontânea prejudicada (77,3\%); Conforto prejudicado (68,3\%); Risco de perfusão cerebral ineficaz (66\%); Risco de choque $(63,8 \%)$; Débito cardíaco diminuído (59,1\%); Troca de gases prejudicada $(52,3 \%)$; e Capacidade adaptativa craniana $(52,3 \%)$. Os autores ressaltam que todos os pacientes que vieram a óbito, apresentaram um ou mais de um destes diagnósticos ${ }^{(5)}$.

Dos diagnósticos identificados nas publicações avaliadas, o principal foi o Risco para infecção. A vítima de trauma está exposta a diversos fatores de risco que podem levar a um quadro de infecção, como procedimentos invasivos realizados muitas vezes dentro da viatura, trauma e exposição ambiental aumentada a patógenos. Autores corroboram que esse achado aponta para a necessidade de medidas de segurança nas intervenções invasivas no setor de emergência e no atendimento pré-hospitalar ${ }^{(6)}$.

Destaca-se que a necessidade de rapidez nos procedimentos que é exigida da equipe multiprofissional, por ocasião do atendimento, não diminui a importância para que estes sejam realizados respeitando os preceitos técnicos de assepsia e antissepsia, à medida que o desacato a esses preceitos tem repercussões negativas na evolução do paciente, sobretudo perante a fragilidade da condição vital das vítimas.

Aidentificaçãodosdiagnósticosdeenfermagem das vítimas de trauma possibilita aos enfermeiros detectar e controlar os riscos precocemente e planejar individualmente o cuidado prestado, por meio de intervenções específicas fundamentadas em evidências científicas. Desta forma, as ações são mais eficazes e imediatas ${ }^{(3)}$.

Destaca-se, ainda como tecnologia levedura a elaboração de protocolos. Estudo recente objetivou a apresentação de um protocolo de cuidados de enfermagem na préremoção aeroespacial de pacientes adultos vítimas de trauma, no intuito de proporcionar a prática de cuidado mais segura aos pacientes aerorremovidos $^{(24)}$. Verifica-se, portanto, que a utilização de protocolos de cuidados se torna um 
importante instrumento na tomada de decisão do enfermeiro, por priorizar e organizar as ações de enfermagem.

\section{Tecnologias de cuidado duras}

As tecnologias duras abrangem os materiais, equipamentos, máquinas e demais instrumentais utilizados durante a realização do cuidado $^{(8)}$. A busca dos artigos evidenciou dois estudos que envolviam destas tecnologias na assistência ao paciente politraumatizado.

Para otimização da assistência e por o trauma ser uma situação clínica emergencial, o auxílio à tecnologia revela-se de grande valia para os profissionais. O uso de sistemas de informações tem se destacado na área da saúde, pois auxilia na tomada de decisões de diagnósticos e de terapêutica nos cuidados ao paciente ${ }^{(25-26)}$.

Pesquisa recente objetivou o desenvolvimento de um sistema de auxílio à tomada de decisão sobre os diagnósticos de enfermagem em vítimas de trauma no atendimento avançado pré-hospitalar móvel, considerando a Taxonomia North American Nursing Diagnosis Association (NANDA), e propor intervenção baseando-se na Nursing Interventions Classification (NIC). Os autores verificaram que o gerenciamento, o armazenamento e o tempo de retorno das informações ocorreram em tempo viável, demonstrando otimização para elaboração dos diagnósticos ${ }^{(25)}$.

Outro estudo buscou identificar diagnósticos e intervenções de enfermagem baseados na Classificação Internacional para a Prática de Enfermagem $\left(\mathrm{CIPE}^{\circledast}\right)$, uma tecnologia de informação. Os autores constataram que o sistema tecnológico permitiu a identificação de diagnósticos e intervenções de enfermagem mais direcionados e sistematizado, além de favorecer a documentação da enfermagem ${ }^{(26)}$.

Desta forma, pode-se inferir que a implementação de sistemas permite cuidado diferenciado por proporcionar redução de tempo na identificação dos diagnósticos de enfermagem, tendo em vista a dinamização e a facilitação do uso das taxonomias NANDA e NIC.

\section{CONSIDERAÇÕES FINAIS}

O trauma é percebido por muitos estudiosos como um evento devastador da sociedade moderna, posto que se constitui em uma epidemia silenciosa e letal. Caracteriza-se, portanto, como um problema de saúde pública, causando forte impacto na morbidade e mortalidade da população.

Inúmeros estudos relatam incorporações de tecnologias do cuidado na prática assistencial de enfermagem ao paciente politraumatizado e esta revisão permitiu comprovar tais fatos. Observouse, nos artigos selecionados, a preocupação dos enfermeiros em prestar assistência mais direcionada, eficaz e imediata ao paciente vítima de trauma, por meio de levantamento de diagnósticos específicos, porém não houve relatos de formulários próprios elaborados pelos enfermeiros especificamente para esse fim.

Nesse interim, se identificou que os profissionais da enfermagem utilizam os três tipos de tecnologia do cuidado, com ênfase às tecnologias leve-duras. Não há dúvidas quanto à melhoria assistencial proporcionada pelas tecnologias do cuidado, visto que esta abrange todos os aspectos do cuidar.

Percebeu-se, então, a necessidade urgente da realização de novas pesquisas ao que diz respeito à sistematização da assistência de enfermagem aos pacientes vítimas de trauma, pois o enfermeiro é um dos principais responsáveis no atendimento inicial desses pacientes e necessita de ferramentas que potencializem este cuidado. Dessa forma, trazem-se benefícios à população, ao profissional e ao sistema, tanto no que diz respeito à qualidade do serviço prestado quanto aos setores econômicos e organizacionais. Ressalta-se, também, a utilização de sistemas de informação para agilizar o processo do cuidar.

A partir dos resultados deste estudo, compreendeu-se ser necessário intensificar esforços para a elaboração de pesquisas sobre o tema investigado, principalmente acerca da prática da sistematização da assistência de enfermagem embasada em formulários próprios para direcionar suas ações.

\section{REFERÊNCIAS}

1. Mattos LM, Silvério MR. Avaliação do indivíduo vítima de politraumatismo pela equipe de enfermagem em um serviço de emergência de Santa Catarina. Rev bras promoç saúde. [Internet] 2012; 25(2) [acesso em 04 jul 2014]. Disponível: http://www.redalyc.org/ pdf/408/40823359008.pdf

2. Martins CBG, Matos KF. Mortalidade por causas acidentais na população infanto-juvenil. Rev Baiana Enferm. [Internet] 2013; 27(2) [acesso em 03 jul 2014]. Disponível: http://www.portalseer.ufba.br/index.php/ enfermagem/article/viewFile/8294/7156 
3. Cyrillo RMZ, Dalri MCB, Canini SRMS, Carvalho EC, Lourencini RR. Diagnósticos de enfermagem em vítimas de trauma atendidas em um serviço préhospitalar avançado móvel. Rev Eletr Enferm. [Internet] 2009; 11(4) [acesso em 04 jul 2014]. Disponível: https:// www.fen.ufg.br/fen_revista/v11/n4/pdf/v11n4a06.pdf

4. Quintero MT, Gómez M. El cuidado de enfermería significa ayuda. Aquichan. [Internet] 2010; 10(1) [acesso em 04 jul 2014]. Disponível: http://www.redalyc.org/ articulo.oa? $\mathrm{id}=74116244002$

5. Sallum AMC, Santos JLF, Lima FD. Diagnósticos de enfermagem em vítimas fatais decorrentes de trauma no cenário da emergência. Rev Latino-Am Enferm. [Internet] 2012; 20(1) [acesso em 02 jul 2014]. Disponível: http://dx.doi.org/10.1590/S0104-11692012000100002

6. Sallum AMC, Sousa RMC. Diagnósticos de enfermagem em vítimas de trauma nas primeiras seis horas após o evento. Acta Paul Enferm. [Internet] 2012; 25(2) [acesso em 02 jul 2014] Disponível: http://dx.doi. org/10.1590/S0103-21002012000200016

7. Nietsche EA, Lima MGR, Rodrigues MGS, Teixeira JA, Oliveira BNB, Motta CA, et al. Tecnologias inovadoras do cuidado de enfermagem. Rev Enferm UFSM. [Internet] 2012; 2(1) [acesso em 02 jul 2014]. Disponível: http://cascavel.ufsm.br/revistas/ojs-2.2.2/index.php/ reufsm/article/view/3591/3144

8. Thofehrn MB; Montesinos MJL, Arrieira IC, Ávila VC, Vasques TCS, Farias ID. Processo de trabalho dos enfermeiros de um hospital da Espanha: ênfase nas tecnologias de cuidado. Cogitare Enferm. [Internet] 2014; 19(1) [acesso em 01 jul 2014]. Disponível: http:// dx.doi.org/10.5380/ce.v19i1.35972

9. Silva FC, da Silva RCL. O enfermeiro e as práticas assistências para o cliente politraumatizado no setor de emergência. Rev Enferm UFPE [Internet]. 2009; 3(4) [acesso em 04 jul 2014]. Disponível: http://www.revista. ufpe.br/revistaenfermagem/index.php/revista/article/ download/92/2917

10. de Souza MT, da Silva MD, de Carvalho R, Marcela T. Revisão integrativa: o que é e como fazer. Einstein. [Internet] 2010; 8(1) [acesso em 02 jul 2014]. Disponível: http://astresmetodologias.com/material/O_que_e_ RIL.pdf

11. Howick J, Chalmers I, Glasziou P, Greenhalgh T, Heneghan C, Liberati A, et al. The Oxford Levels of Evidence 2. [Internet]. Oxford Centre for EvidenceBased Medicine The Oxford 2011 Levels of Evidence; 2011 [atualizada em 2014; acesso em 29 de jul 2014]. Disponível: http://www.cebm.net/ocebm-levels-ofevidence

12. Pinto ACS, Luna IT, Silva AA, Pinheiro PNC, Braga VAB, Souza AMA. Fatores de risco associados a problemas de saúde mental em adolescentes: revisão integrativa. Rev Esc Enferm USP. [Internet] 2014; 48(3) [acesso em 02 jul 2014]. Disponível: http://dx.doi. org/10.1590/S0080-623420140000300022

13. Grilo CM, Rocha JMC. As vivências do indivíduo perante uma situação de trauma em contexto de urgência. Rev Enferm UFPE [Internet]. 2011; 5(8) [acesso em 01 jul 2014]. Disponível: http://www.revista. ufpe.br/revistaenfermagem/index.php/revista/article/ download/2091/2364

14. Flores YYR, Flores MMH, Rangel MG. Enseñar a quien cuida el arte de cuidar: un programa educativo con cuidadores de pacientes politraumatizados. Invest. Educ. Enferm. [Internet] 2010; 28(1) [acesso em 01 jul 2014]. Disponível: http://www.redalyc.org/articulo. oa?id=105215294010

15. Divino EA, Pereira QLC, Siqueira HCH. A capacitação da equipe que atua no atendimento pré-hospitalar móvel: necessidade e importância da educação permanente na perspectiva dos trabalhadores. Rev Min Enferm. [Internet]. 2009; 13(3) [acesso em 01 jul 2014]. Disponível: http://www.enf.ufmg.br/site_novo/ modules/mastop_publish/files/files_4c0e4bd3d5213. pdf

16. Bueno AA, Bernardes A. Percepção da equipe de enfermagem de um serviço de atendimento pré-hospitalar móvel sobre o gerenciamento de enfermagem. Texto Contexto Enferm. [Internet] 2010; 19(1) [acesso em 03 jul 2014]. Disponível: http://dx.doi. org/10.1590/S0104-07072010000100005

17. Santos JLG, Lima MADS, Pestana AL, Garlet ER, Erdmann AL. Desafios para a gerência do cuidado em emergência na perspectiva de enfermeiros. Acta Paul Enferm. [Internet] 2013; 26(2): 136-143 [acesso em 13 jul 2014]. Disponível: http://dx.doi.org/10.1590/S010321002013000200006

18. Oliveira JM. Diagnósticos e intervenções de enfermagem em vítimas de trauma durante atendimento pré-hospitalar utilizando a CIPE ${ }^{\circledR}$. Rev Eletr Enferm [Internet]. 2013; 15(1). [acesso em 12 jul 2014]. Disponível: http://www.fen.ufg.br/fen_revista/ v15/n1/pdf/v15n1a04.pdf

19. Nascimento ERP, Hilsendeger BR, Neth C, Belaver GM, Bertoncello KCG. Classificação de risco na emergência: avaliação da equipe de enfermagem. Rev Enferm UERJ. [Internet] 2011; 19(1) [acesso em 12 jul 2014]. Disponível: http://www.facenf.uerj.br/v19n1/ v19n1a14.pdf

20. Nascimento ERP, Hilsendeger BR, Neth C, Belaver GM, Bertoncello KCG. Acolhimento com classificação de risco: avaliação dos profissionais de enfermagem de um serviço de emergência. Rev Eletr Enferm. [Internet] 2011; 13(4) [acesso em 12 jul 2014]. Disponível: https:// www.fen.ufg.br/fen_revista/v13/n4/pdf/v13n4a02.pdf 
21. Júnior JAB, Matsuda LM. Acolhimento com classificação de risco em serviço hospitalar de emergência: avaliação da equipe de enfermagem. Rev Min Enferm. [Internet] 2012; 16(3) [acesso em 03 jul 2014]. Disponível: http://www.reme.org.br/artigo/ detalhes/545

22. Ribeiro NCA, Barreto SCC, Hora EC, Sousa RMC. O enfermeiro no cuidado à vítima de trauma com dor: o quinto sinal vital. Rev Esc Enferm USP. [Internet] 2011; 11(1) [acesso em 01 jul 2014]. Disponível: http://dx.doi. org/10.1590/S0080-62342011000100020

23. Romanek FARM, Avelar MCQ. A multidimensionalidade da dor no ensino de enfermagem em atendimento pré-hospitalar, às vítimas de trauma. Rev Dor. [Internet] 2012; 18(4) [acesso em 04 jul 2014]. Disponível: http://dx.doi.org/10.1590/S180600132012000400008

24. Schweitzer G, Nascimento ERP, Moreira AR, Bertoncello KCG. Protocolo de cuidados de enfermagem no ambiente aeroespacial a pacientes traumatizados: cuidados antes do voo. Rev Bras Enferm. [Internet] 2011; 64(6) [acesso em 13 jul 2014]. Disponível: http://dx.doi.org/10.1590/S0034-71672011000600011

25. Caritá EC, Nini RA, Melo AS. Sistema de auxílio aos diagnósticos de enfermagem para vítimas de trauma no atendimento avançado pré-hospitalar móvel utilizando as Taxonomias NANDA e NIC. J Health Inform. [Internet] 2010; 2(4) [acesso em 04 jul 2014]. Disponível: http://www.jhi-sbis.saude.ws/ojs-jhi/ index.php/jhi-sbis/article/view/108/36

26. Lins TH, Lima AAXBC, Verrísimo RCSS, Oliveira JM. Diagnósticos e intervenções de enfermagem em vítimas de trauma durante atendimento pré-hospitalar utilizando a CIPE ${ }^{\oplus}$. Rev Eletr Enferm [Internet]. 2013; 15(1) [acesso em 04 jul 2014]. Disponível: http://www. fen.ufg.br/fen_revista/v15/n1/pdf/v15n1a04.pdf 